\title{
High resolution atomic coherent control via spectral phase manipulation of an optical frequency comb
}

\author{
Matthew C. Stowe, Flavio C. Cruz,* and Jun Ye \\ JILA, National Institute of Standards and Technology and University of Colorado \\ and Department of Physics, University of Colorado, Boulder, CO 80309-0440
}

(Dated: July 2, 2018)

\begin{abstract}
We demonstrate high resolution coherent control of cold atomic Rubidium utilizing spectral phase manipulation of a femtosecond optical frequency comb. Transient coherent accumulation is directly manifested by the enhancement of signal amplitude and spectral resolution via the pulse number. The combination of frequency comb technology and spectral phase manipulation enables coherent control techniques to enter a new regime with natural linewidth resolutions.

PACS numbers: 32.80.Qk, 32.80-t, 39.30.+w, 39.25.+k
\end{abstract}

The introduction of phase-stabilized optical frequency combs has revolutionized the field of precision atomic and molecular spectroscopy [1, 2]. In our previous work we used direct frequency comb spectroscopy (DFCS) to demonstrate high resolution measurements of one- and two-photon transitions in cold ${ }^{87} \mathrm{Rb}[3,4]$. This new technique has the significant advantage that the comb frequencies may be absolutely referenced, for example to a Cesium atomic clock, enabling precision spectroscopy over a bandwidth of several tens of nanometers. The time-domain phase coherence offered by the optical frequency comb has stimulated research in areas of atomic clocks [5, [] , optical and radio frequency transfer [7], and more recently high harmonic generation [8, 9]. In parallel, the field of coherent control of atomic and molecular systems has seen advances incorporating high power femtosecond laser sources and pulse shaping technology. This has allowed for demonstrations of robust coherent population transfer via adiabatic passage techniques 10, 11], coherent control of two-photon absorption 12, 13, 14], resolution enhancement of coherent anti-Raman scattering 15], and progress towards cold atom photoassociation [16]. It is under this exciting context that we combine the femtosecond comb and spectral phase manipulation with the aim towards coherent control at the highest possible spectroscopic resolution.

In this Letter, properties of the transient coherent accumulation effect, including spectral phase manipulation, are explicitly demonstrated via a finite number of phasestabilized femtosecond pulses. Specifically, we measure atomic transition linewidth and population transfer versus the number of applied phase-coherent femtosecond pulses and as a function of linear frequency chirp. The basic idea of multi-pulse coherent accumulation is that the amplitude for excitation of a specific atomic energy level may be increased significantly with the number of femtosecond pulses, and can be done such that other non-resonant states remain unexcited, thus enabling high state selectivity. We show that tuning the comb leads to destructive quantum interference of the two-photon transition amplitude only observable in this multi-pulse context and may be useful for eliminating nonlinear absorption. Positive and negative linear frequency chirp is then applied to the comb modes and thus the two-photon transition amplitudes. It is shown that the role of chirp in the single pulse case is no longer necessarily applicable in these multi-pulse experiments since the atomic coherence persists between femtosecond pulses. The combination of pulse shaping with the femtosecond comb is shown to increase the signal of a two-photon transition at a specific chirp while maintaining high resolution.

The spectrum of a train of femtosecond pulses is a set of approximately $10^{6}$ comb modes equally spaced by the repetition frequency $\left(f_{r}\right)$ of the mode-locked laser. The frequency of each comb mode is given by the relation $\nu_{N}=f_{o}+N \times f_{r}$, where $f_{o}$ is the carrier-envelope offset frequency and $N$ is an integer mode number of the order $10^{6}$ [2]. The frequencies of the comb modes are independent of the spectral phase provided all pulses in the train have the same spectral phase. For the two-photon transitions we investigate, there are of the order $2 N$ different transition amplitudes from the ground to the excited state. It is instructive to consider the frequency spectrum of the laser field consisting of a finite number of pulses. In this work we use from one to approximately 100 pulses for atomic excitation. The linewidth of the laser field after a single pulse is approximately $30 \mathrm{~nm}$, and after two pulses the spectrum is sinusoidally modulated with a spacing of $f_{r}$ between peaks. In general, a comb mode lineshape is described by a $\operatorname{sinc}^{2}$ function of the pulse number. As the linewidth narrows, the peak power of any comb mode grows as the number of pulses squared. This amplitude and spectral resolution enhancement presents a clear distinction from previous experiments employing single pulse excitation.

To explore the transient coherent accumulation effects, we use a sample of ${ }^{87} \mathrm{Rb}$ atoms laser cooled and trapped in a magneto-optic trap (MOT). This allows for approximately 50 pulses to coherently interact with the atomic system via the $5 S_{1 / 2} \mathrm{~F}=2$ to $5 P_{3 / 2} \mathrm{~F}=3$ to $5 D_{5 / 2} \mathrm{~F}=4$ two-photon transition (Fig. 1(a)). We use a Kerr lens mode locked Ti:Sapphire laser which operates at a center wavelength of $778 \mathrm{~nm}$ with a $30 \mathrm{~nm}$ wide spectrum and $100 \mathrm{MHz}$ repetition rate. This produces transform limited pulses of approximately 40 fs duration. $f_{o}$ is measured via the typical $f-2 f$ interferometer technique and 


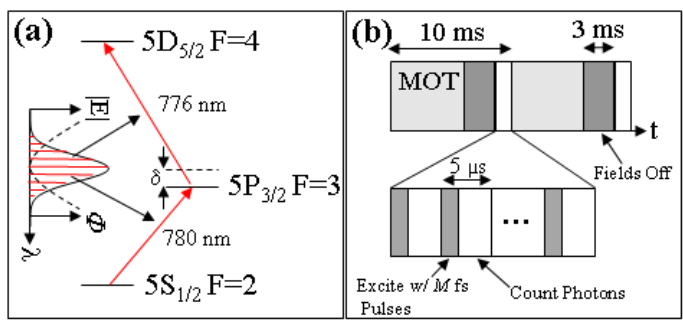

FIG. 1: (color online) (a) Simplified energy level diagram of the three relevant atomic states and frequency domain representation of the chirped comb. $\delta$ is the detuning of the intermediate state from half the two-photon transition frequency. (b) Timing diagram used in the experiments.

locked to a stable radio frequency (rf) source which is fixed at a prescribed value or scanned. $f_{r}$ is stabilized via a Cs-referenced low phase-noise rf source and steered so as to maintain the appropriate absolute frequency of the comb modes. In the following experiments, $f_{r}$ is locked to $100.41356730 \mathrm{MHz}$, which for $f_{o}$ values of either 18.14 $\mathrm{MHz}$ or $-31.86 \mathrm{MHz}$ ensures two-photon resonance between the $5 S_{1 / 2} \mathrm{~F}=2$ ground state and $5 D_{5 / 2} \mathrm{~F}=4$. For $f_{o}=18.14 \mathrm{MHz}$, there is a comb mode resonant with the intermediate state $5 P_{3 / 2} \mathrm{~F}=3$. In the other case of $f_{o}=$ $-31.86 \mathrm{MHz}$, no mode is resonant with $5 P_{3 / 2} \mathrm{~F}=3$, but rather all modes are symmetrically detuned around this intermediate resonance. The signal is proportional to the $5 \mathrm{D}$ population and is measured by photon counting the $420 \mathrm{~nm}$ 5D-6P-5S cascade fluorescence. For appropriately chosen values of $f_{r}$ and $f_{o}$, the signal after a sufficient number of pulses to reach steady state excitation can be attributed to only this $5 D_{5 / 2} \mathrm{~F}=4$ resonant hyperfine level [3]. After many pulses all other $5 \mathrm{D}$ and $7 \mathrm{~S}$ hyperfine states, although covered by the $30 \mathrm{~nm}$ comb spectrum, are detuned many linewidths away from the closest comb modes and as a result remain unexcited.

The theory model describing the experimental results is based on numerical solution of the Liouville equation for the density matrix of the three level atomic system. Decoherence of the electronic system is included via phenomenological decay terms due to the natural linewidth of $6 \mathrm{MHz}$ for the $5 \mathrm{P}$ and $660 \mathrm{kHz}$ for the $5 \mathrm{D}$ atomic states. The driving field is taken to be a linearly chirped pulse of $30 \mathrm{~nm}$ bandwidth at $778 \mathrm{~nm}$ and a transform limited peak field strength of $E_{o}=10^{7} \mathrm{~V} / \mathrm{m}$. The solution of the Liouville equation is stepped through time via a fourth order Runge-Kutta algorithm. After numerical solution for a single pulse, the density matrix is advanced in time by approximately $1 / f_{r}$ to the next pulse analytically, then repeated for an arbitrary number of pulses. This technique differs from some previous work on modeling two-photon coherent control with femtosecond pulses as it includes effects of atomic decoherence and the impulsive excitation approximation is not used [14, 17]. Our model includes only the $5 S_{1 / 2} \mathrm{~F}=2,5 P_{3 / 2}$ $\mathrm{F}=3,5 D_{5 / 2} \mathrm{~F}=4$ hyperfine levels. Because the comb is tuned on resonance with this transition, the three-level model is a good approximation to our experiment after several pulses. However, for excitation by less than a few pulses the other hyperfine states can not necessarily be considered off-resonance because the frequency comb structure of the field is not manifest.

The data acquisition cycle (Fig. 1(b)) consists of loading the MOT, shutting off all MOT-related fields, then exciting the free cold atoms. A pockels cell with $8 \mathrm{~ns}$ rise time is used as a fast shutter to transmit any desired number of pulses to the atoms. The triggering for the pockels cell is directly from the laser repetition frequency to ensure that the pockels cell is always fully on or off when a femtosecond pulse is incident. Tunable linear frequency chirp is generated by a double pass grating stretcher and compressor which allows for chirp up to $\phi^{\prime \prime}= \pm 2.5 \times 10^{5} f s^{2}$, defined as $\phi^{\prime \prime}=d \phi^{2} / d \omega^{2}$. Some higher order dispersion is present, but our data is generally well modeled by the inclusion of only quadratic spectral phase. The MOT is loaded for $6 \mathrm{~ms}$, then the magnetic field is turned off for $3 \mathrm{~ms}$ during which time an optical molasses remains, finally the atoms are probed after all other fields are off. The timing diagram in (Fig. 1(b)) shows the atoms are excited by $M$ pulses, spontaneously emitted photons are counted for $5 \mu \mathrm{s}$ as the atoms relax, then repeated 40 times per MOT.

We first demonstrate the coherent accumulation of a controlled number of femtosecond pulses on the $5 D_{5 / 2}$ $\mathrm{F}=4$ population and lineshape. Previous work on DFCS [3, 4] was conducted only under steady state atomic excitation, i.e. excitation times longer than the atomic coherence time. This is necessary for spectroscopy with the highest possible resolution, but we investigate properties of the transient femtosecond coherent accumulation for pulse numbers, or equivalently excitation times significantly less than the $240 \mathrm{~ns} 5 D_{5 / 2} \mathrm{~F}=4$ atomic lifetime. The $f_{r}$ and $f_{o}$ of the comb are tuned to 100.41356730 $\mathrm{MHz}$ and $18.14 \mathrm{MHz}$ respectively, which ensure that a comb mode is resonant with the $5 S_{1 / 2} \mathrm{~F}=2$ to $5 P_{3 / 2}$ $\mathrm{F}=3$ one-photon transition and another with the $5 P_{3 / 2}$ $\mathrm{F}=3$ to $5 D_{5 / 2} \mathrm{~F}=4$ transition (Fig. 2 inset). We split the probe laser and counter-propagate the two intensitymatched beams through the atoms to balance the radiation pressure and thus minimize any line broadening due to accumulated Doppler shifts. For the chirped excitation experiments described later the laser is not counter-propagated. The femtosecond probe laser is focused with linear polarization to a diameter of $160 \mu \mathrm{m}$ into the MOT, resulting in an electric field strength per beam of approximately $10^{7} \mathrm{~V} / \mathrm{m}$. Figure 2 shows the total number of photon counts (in squares) that occur after the $M^{t h}$ pulse and is a measure proportional to the total 5D population after excitation by $M$ pulses. The dashed line in Fig. 2 is the result from our theory model for low power multi-pulse excitation such that there is no power broadening of the linewidth. In the actual experiment the pulse intensity is high enough to excite a significant $5 \mathrm{D}$ population and it saturates to its maximum value at a smaller number of pulses than in the case of weak 


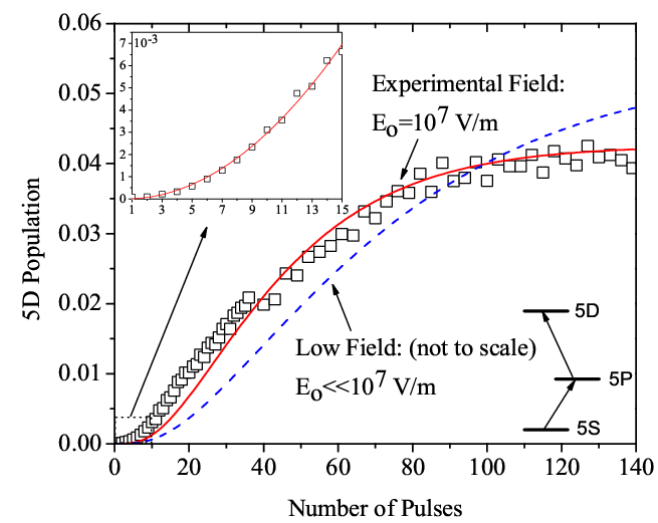

FIG. 2: (color online) The on-resonance $5 D_{5 / 2} \mathrm{~F}=4$ fluorescence signal is measured (squares) at a field strength of approximately $E_{o}=10^{7} \mathrm{~V} / \mathrm{m}$ versus the number of applied pulses. The solid (dashed) line is the theoretically predicted excited state populations under experimental (asymptotically low) field strengths. The inset shows the quadratic scaling for the first 15 pulses.

excitation (Fig. 2). This is the time domain picture of power broadening of the transition linewidth due to the femtosecond probe laser. For pulse numbers significantly smaller than the $5 \mathrm{D}$ coherence time the population scaling can be fit well by a second order polynomial in the pulse number or equivalently the accumulated area (inset Fig. 2). This coherent enhancement of the population versus pulse number demonstrates the increased efficiency compared to excitation by a single pulse of the same total power for the case of two-photon absorption via a resonant intermediate state.

The coherent accumulation that enables the large enhancement of the resonant 5D population simultaneously enables the high resolution necessary for exciting a single 5D hyperfine level. In this part of the experiment the lineshape is measured by scanning $f_{o}$ after excitation from $M$ pulses to directly demonstrate the resolution enhancement. As was mentioned previously the spectral content of the field generated by a finite number of pulses is a series of modes each with a linewidth inversely proportional to the number of pulses coherently accumulated. Figure 3 illustrates this effect through the lineshape and width of the measured $5 D_{5 / 2} \mathrm{~F}=4$ resonance after excitation by $10,15,20$, and 80 pulses. The asymptotic power broadened linewidth of $2 \mathrm{MHz}$ occurs at 80 pulses which is approximately the number of pulses the $5 D_{5 / 2} \mathrm{~F}=4$ population in Fig. 2 becomes saturated.

Some of the original work on coherent control has demonstrated the modification of the quantum interference of the two-photon transition in $\mathrm{Rb}$ and $\mathrm{Cs}$ by shaping a single amplified femtosecond pulse [12, 13, 14]. It was shown that for the transition from $5 \mathrm{~S}$ to $5 \mathrm{D}$ in $\mathrm{Rb}$ the excited state population was essentially zero for large negative chirp, where higher frequencies precede lower (see the single pulse theory in Fig. 4). For low field strengths (in the absence of adiabatic following effects

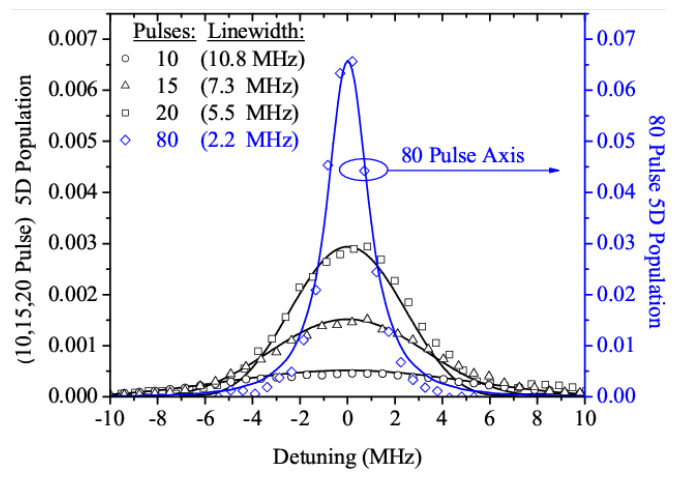

FIG. 3: (color online) The $5 D_{5 / 2} \mathrm{~F}=4$ lineshape is measured (symbols) versus the number of applied femtosecond pulses $(10,15,20,80$ pulses) with the corresponding FWHM linewidth in the legend. The Lorenztian linewidth of $2.2 \mathrm{MHz}$ after 80 pulses is the asymptotic power broadened linewidth.

[10, 11]) and large negative chirps, this can be understood conceptually as the front of the pulse is only resonant with the $5 \mathrm{P}$ to $5 \mathrm{D}$ transition. Due to the fact that there is no initial population in $5 \mathrm{P}$ a resonantly enhanced two-photon transition can not occur, the only contribution can be from the frequencies corresponding to approximately half the 5S-5D energy splitting. Similar to previous experiments with single pulse excitation we observe a characteristic oscillation of $5 \mathrm{D}$ population versus chirp with a period given by $\phi_{2 \pi}^{\prime \prime}=2 \pi / \delta^{2}[13]$; the definition of $\delta$ is given in Fig. 1(a). The measured period of $\phi_{2 \pi}^{\prime \prime}=14.5 \times 10^{4} f s^{2}$ agrees well with the theory value of $\phi_{2 \pi}^{\prime \prime}=14.3 \times 10^{4} f s^{2}$ for $5 P_{3 / 2} \mathrm{~F}=3$. However, for negative chirps significantly different results are observed for multi-pulse excitation as shown in Fig. 4. This difference is due to the $5 \mathrm{~S}-5 \mathrm{P}$ electronic coherence that

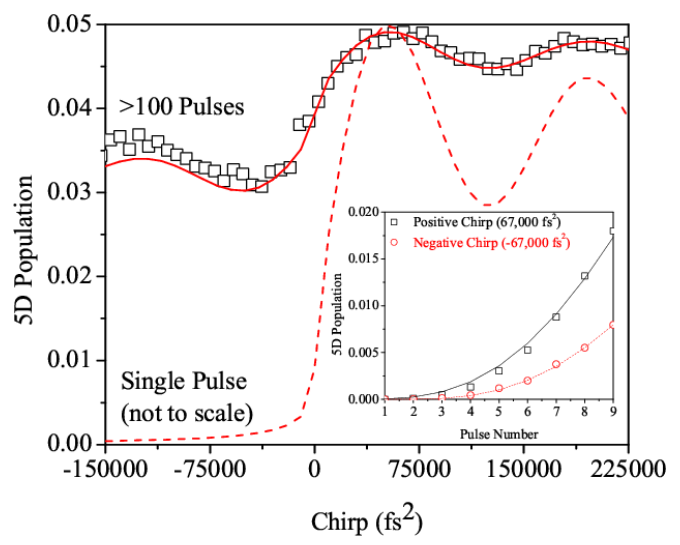

FIG. 4: (color online) The $5 D_{5 / 2} \mathrm{~F}=4$ excited state fluorescence is measured (squares) under steady state excitation ( $>100$ pulses) versus the applied linear frequency chirp. The solid line indicates the steady state theory and the dashed line is the single pulse theory. Inset: The signal (symbols) versus pulse number for two values of equal and opposite chirp, the solid and dashed lines are theoretical predictions. 


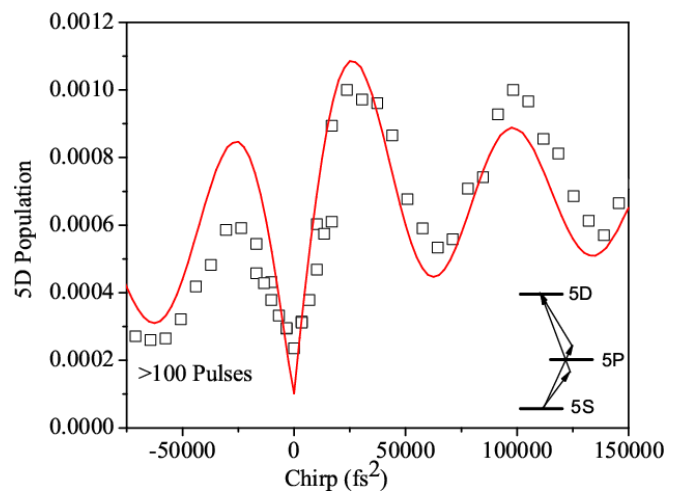

FIG. 5: (color online) Measured fluorescence (squares) and theoretical prediction (solid line) for the case of destructive quantum interference versus linear frequency chirp. The inset shows two of the many two-photon resonant comb mode pairs symmetrically detuned from the intermediate $5 P_{3 / 2} \mathrm{~F}=3$ resonance, the closest pair is $\pm 50 \mathrm{MHz}\left( \pm f_{r} / 2\right)$ detuned.

persists for approximately $50 \mathrm{~ns}$, or about five inter-pulse periods. For negative-chirp pulses the intermediate state can be excited by the low frequency end of the preceding pulse and the high frequency beginning of the next pulse can complete the two-photon transition. Figure 4 inset shows the $5 \mathrm{D}$ population at $\phi^{\prime \prime}= \pm 6.7 \times 10^{4} \mathrm{fs}^{2}$ versus the number of pulses to illustrate that the scaling of $5 \mathrm{D}$ population is smaller for negative chirp. We emphasize that unlike single pulse experiments our results involve only three well resolved hyperfine levels chosen such that hyperfine selection rules prohibit any transitions from other states to $5 D_{5 / 2} \mathrm{~F}=4$. It is for this reason that we may be attribute the measured oscillation period, $\phi_{2 \pi}^{\prime \prime}$, to only one intermediate state. This contrasts previous work where the $5 P_{1 / 2}$ states exhibit a shorter period, $\phi_{2 \pi}^{\prime \prime}=2.38 \times 10^{3} \mathrm{fs}^{2}$, oscillation superposed on the larger $5 P_{3 / 2}$ signal [11, 14].

Our final experiment further demonstrates the versatility of the high resolution frequency comb with spectral phase control by significantly reducing the nonlinear absorption. The phase of the two-photon transition ampli- tude for a pair of modes is a function of the intermediate state detuning [18]. The detuning of the closest mode to the intermediate $5 P_{3 / 2} \mathrm{~F}=3$ state can be tuned from $0 \mathrm{MHz}$ to at most $f_{r} / 2$. In the case of all modes symmetrically detuned around $5 P_{3 / 2} \mathrm{~F}=3$, the relative phase between the $\pm 50 \mathrm{MHz}$ detuned two-photon transition amplitudes is $2 \times \tan ^{-1}(2 \times(50 \mathrm{MHz} / 6 \mathrm{MHz})) \simeq 173^{0}$, between all other further detuned mode pairs it is $\simeq 180^{\circ}$. This phase flip around the intermediate state results in nearly complete destructive quantum interference of the two-photon transition probability for transform limited pulses [12]. Translating the entire comb by varying $f_{o}$ can easily position all mode pairs symmetrically around the intermediate state, while maintaining two photon resonance (Fig. 5, inset). As shown in Fig. 5, this results in approximately 50 times less $5 \mathrm{D}$ population than the previous case when a mode is tuned on $5 P_{3 / 2} \mathrm{~F}=3$ resonance. Linear frequency chirp introduces extra relative phase between amplitudes detuned above and below the $5 \mathrm{P}$ resonance and reduces the net destructive interference. As shown in Fig. 5, the two-photon transition probability is modulated versus chirp, with a minimum $5 \mathrm{D}$ population at zero chirp.

In conclusion, we have demonstrated transient coherent accumulation via a controlled number of phasecoherent femtosecond pulses in an atomic system. The application of spectral phase manipulation to this multipulse regime enables fundamental coherent control experiments to be performed at a resolution limited only by natural resonance widths. The present work is a step towards combining pulse shaping from the field of coherent control with femtosecond comb technology. This research is expected to enable high resolution coherent control, for example, in wavepacket shaping for molecular photo-association [19].

We thank funding support from ONR, NSF, and NIST. We gratefully thank A. Marian for the work on steadystate DFCS.

*Permanent address: Instituto de Fisica Gleb Wataghin, UNICAMP, Campinas, SP, 13083-970, Brazil.
[1] Th. Udem, R. Holzworth, and T. W. Hansch, Nature 416, 233 (1999).

[2] S. T. Cundiff and J. Ye, Rev. Mod. Phys. 75, 325 (2003).

[3] A. Marian et al., Science 306, 2063 (2004).

[4] A. Marian et al., Phys. Rev. Lett. 95, 023001 (2005).

[5] S. A. Diddams et al., Science 293, 825 (2001).

[6] J. Ye, L.-S. Ma, and J. L. Hall, Phys. Rev. Lett. 87, 270801 (2001).

[7] K. W. Holman et al., Opt. Lett. 30, 1225 (2005).

[8] R. J. Jones et al., Phys. Rev. Lett. 94, 193201 (2005).

[9] C. Gohle et al., Nature 436, 234 (2005).

[10] J. R. Kuklinski et al., Phys. Rev. A 40, 6741 (1989).

[11] B. Broers, H. B. van Linden van den Heuvell, and L. D.
Noordam, Phys. Rev. Lett. 69, 2062 (1992).

[12] D. Meshulach and Y. Silberberg, Nature 396, 239 (1998).

[13] P. Balling, D. J. Maas, and L. D. Noordam, Phys. Rev. A 50, 4276 (1994).

[14] B. Chatel et al., Phys. Rev. A 68, 041402 (2003).

[15] D. Oron et al., Phys. Rev. Lett. 88, 063004 (2002).

[16] W. Salzmann et al., arXiv:physics/0509056 1 (2005).

[17] D. Felinto, L. H. Acioli, and S. S. Vianna, Phys. Rev. A 70, 043403 (2004).

[18] T. H. Yoon et al., Phys. Rev. A 63, 011402 (2000).

[19] J. Vala et al., Phys. Rev. A 63, 013412 (2000). 